\section{W. K. $\mathrm{TSO}^{1}$ \\ Senior Engineer, \\ Northrop Corporation, \\ Norair Division, \\ Hawthorne, Calif.}

\section{T. K. CAUGHEY}

Professor of Applied Mechanics, California Institute of Technology

Pasadena, Calif.

\title{
Parametric Excitation of a Nonlinear System
}

A study has been made on the parametric excitation of a nonlinear system with hardening springs. In the parametric excitation of linear systems, the only subharmonic predicted is a subharmonic of order 1/2. However, in the parametric excitation of nonlinear systems, it is shown that higher order subharmonics can exist, depending both on the amplitude and frequency of the parametric excitation.

$p$ Parametric excitations of linear systems have been studied to some extent $[1,2,3] .{ }^{2}$ Such studies are necessary to investigate the stability of the solution of a nonlinear system under infinitesimal perturbation. The use of the theory of Mathieu functions and the Strutt-Ince chart predicts the existence of a subharmonic of order $1 / 2$. However, no other subharmonics of higher order are predicted from such studies.

This paper is a study of the parametric excitation of a nonlinear system. Consider a nonlinear two-degree-of-freedom system, and assume that the generalized coordinates are chosen so that the two equations of motion are uncoupled if they were linearized. When one generalized coordinate is harmonically excited, the second coordinate is under parametric excitation through the nonlinear coupling terms with the first generalized coordinate. Investigation of the behavior of the second coordinate involves the study of parametric excitation of a nonlinear system. It is shown in this paper that subharmonics of high order can exist under suitable parametric excitations. The study is motivated by some experimental results from a vibrational test of some monosymmetric thin-wall beams of open section $[4,5]$, where subharmonics of order $1 / 14$ and over have been observed.

\section{Statement of Problem}

Consider the following equation:

$\ddot{x}(t)+x(t)+b_{1} x^{m}(t)+b_{2} x^{n-1}(t)-b_{3} x^{n-1}(t) \cos \lambda t=0$

where $b_{1}, b_{2}$, and $b_{3}$ are positive constants, $m$ and $n$ are positive integers, $m$ being odd, $n$ being even, and $m>n$. Equation (1) represents a nonlinear system which is being parametrically excited. The parametric excitation has a frequency of $\lambda$ and amplitude $b_{3}$.

Seeking a subharmonic solution of order $1 / n$, assume a solution of the form

$$
x(t)=B(t) \cos \left[\frac{\lambda}{n} t-\phi(t)\right] \equiv B(t) \cos [\theta(t)]
$$

Substituting equation (2) into equation (1) and requiring

$$
\dot{B} \cos \theta+B \dot{\phi} \sin \theta=0
$$

there is obtained

$$
-\ddot{B} \sin \theta+B \dot{\phi} \cos \theta+\frac{1}{\omega}\left(1-\omega^{2}\right) B \cos \theta
$$

$$
+f(B \cos \theta, t)=0
$$

${ }^{1}$ Formerly, Graduate Student, California Institute of Technology, Pasadena, Calif.

2 Numbers in brackets designate References at end of paper.

Contributed by the Applied Mechanics Division for presentation at the Winter Annual Meeting, Chicago, Ill., November 7-11, 1965, of The American Society of Mechanical Engineers.

Discussion of this paper should be addressed to the Editorial Department, ASME, United Engineering Center, 345 East 47 th Street, New York, N. Y. 10017, and will be accepted until one month after final publication of the paper itself in the JounNar of APpLIED Mechanics. Manuscript received by ASME Applied Mechanics Division, October 5, 1964; revised draft, April 6, 1965. Paper No. 65 -WA/APM-7. where $\quad \omega \equiv \lambda / n$

and

$f(B \cos \theta, t) \equiv \frac{1}{\omega}\left[b_{1}(B \cos \theta)^{m}+b_{2}(B \cos \theta)^{n-1}\right.$

$$
\left.-b_{3}(B \cos \theta)^{n-1} \cos \lambda t\right]
$$

Multiplying equation (3) by $\cos \theta$ and equation (4) by $\sin \theta$, and subtracting, there is obtained

$$
\dot{B}-\frac{1}{\omega}\left(1-\omega^{2}\right) B \cos \theta \sin \theta-f \sin \theta=0
$$

Multiplying equation (3) by $\sin \theta$ and equation (4) by $\cos \theta$, and adding, there is obtained

$$
B \dot{\phi}+\frac{1}{\omega}\left(1-\omega^{2}\right) B \cos ^{2} \theta+f \cos \theta=0
$$

If $B(t)$ and $\phi(t)$ are slowly varying, ${ }^{3}$ the slowly varying parameter technique [6] can be applied to equations (6) and (7). In this application, $B(t)$ and $\phi(t)$ are considered constant over one cycle. Thus, integrating equations (6) and (7) with respect to $\theta$ from zero to $2 \pi$, there is obtained

$$
\dot{B}-\gamma B^{n-1} \sin n \phi=0
$$

and

$$
B \dot{\phi}+\frac{1}{2 \omega}\left(1-\omega^{2}\right) B+\alpha B^{m}+\beta B^{n-1}-\gamma B^{n-1} \cos n \phi=0
$$

where

$$
\begin{gathered}
\alpha \doteq \frac{b_{1}}{2^{m} \omega}{ }^{m} C_{\frac{m-1}{2}}>0 \\
\beta \equiv \frac{b_{2}}{2^{n-1} \omega}{ }^{n-1} C_{\frac{n-2}{2}}>0 \\
\gamma \equiv \frac{b_{3}}{2^{n} \omega}>0
\end{gathered}
$$

and

$$
{ }^{m} C_{n} \equiv \frac{m !}{n !(m-n) !}
$$

Equations (8) and (9) can be combined and written as

${ }^{3}$ It will be observed that $B$ and $\phi$ will be slowly varying if those terms in equations (6) and (7) that do not involve time derivatives can be regarded as small. Alternately, the conditions can be stated that the terms in equations (8) and (9) that do not involve time derivatives can be regarded as small in comparison to $B \omega$; namely

$$
\begin{aligned}
\frac{\gamma B^{n-2}}{\omega} \ll 1, \text { and } \mid \frac{1}{2 \omega^{2}}\left(1-\omega^{2}\right) & +\frac{\alpha}{\omega} B^{m-1} \\
& +\frac{\beta}{\omega} B^{n-2}-\frac{\gamma}{\omega} B^{n-2} \cos n \phi \mid \ll 1
\end{aligned}
$$




$$
\frac{d B}{d \phi}=\frac{-\gamma B^{n} \sin n \phi}{\alpha B^{m}+(\beta-\gamma \cos n \phi) B^{n-1}+\frac{\left(1-\omega^{2}\right)}{2 \omega} B}
$$

Equation (11) is an exact differential, and its first integral is

$$
\frac{\alpha}{m+1} B^{m+1}+\frac{(\beta-\gamma \cos n \phi)}{n} B^{n}+\frac{\left(1-\omega^{2}\right)}{4 \omega} B^{2}=K
$$

where $K$ is a constant of integration. For each value of $K$, equation (12) defines a trajectory in the $B-\phi$ phase plane, using $B$ as the polar distance from the origin and $\phi$ as the polar angle.

For steady-state vibrations, $\dot{B}=0$ and $\dot{\phi}=0$. From equations (8) and (9), there is obtained

$$
\begin{gathered}
\gamma B^{n-1} \sin n \phi=0 \\
\alpha B^{m}+(\beta-\gamma \cos n \phi) B^{n-1}+\frac{\left(1-\omega^{2}\right)}{2 \omega} B=0
\end{gathered}
$$

The singular points in the $B-\phi$ phase plane correspond to the values of $B$ and $\phi$ that satisfy equations (13) and (14) simultaneously. The possible singularities are

(i)

$$
B=0
$$

If $B \neq 0$, then

(ii)

$$
n \phi=0 \text { or } \pi
$$

and

$$
\alpha B^{m-1}+(\beta-\gamma \cos n \phi) B^{n-2}+\frac{\left(1-\omega^{2}\right)}{2 \omega}=0
$$

Only the positive real roots of $B$ in equation (17) are of interest because, being the polar distance in the $B-\phi$ plane, $B$ is never negative. There is no formula to give the roots of equation (17) in terms of $\alpha, \dot{\beta}, \gamma, m$, and $n$ explicitly in general. However, the distribution of positive real roots of equation (17) can be found using Descartes' rule of sign changes in determining the number of real roots of algebraic equations.

A summary of the distribution of positive roots of equation (17) is given in Table 1.

The nature of the singularities can be studied as follows:

Let

$$
\begin{aligned}
& B=B_{0}+\xi \\
& \phi=\phi_{0}+\eta
\end{aligned}
$$

where $B_{0}$ and $\phi_{0}$ give the location of a singularity and $\xi$ and $\eta$ are perturbation variables describing the trajectories in the neighborhood of the singularity in the $B-\phi$ phase plane. Substituting equations (18) and (19) in equations (8) and (9), noting the value of $n \phi_{0}$ is either zero or $\pi$, and retaining only the first power of $\xi$ and $\eta$ there is obtained

$$
\dot{\xi}=\left(n \gamma B_{0}^{n-1} \cos n \phi_{0}\right) \eta
$$

$$
\begin{array}{r}
B_{0} \dot{\eta}=-\left[m \alpha B_{0}{ }^{m-1}+(n-1)\left(\beta-\gamma \cos n \phi_{0}\right) B_{0}{ }^{n-2}\right. \\
\left.+\frac{1-\omega^{2}}{2 \omega}\right] \xi
\end{array}
$$

Using equations (14) and (20), equation (21) becomes

$B_{0} \ddot{\eta}=-\left[(m-1) \alpha{\overline{B_{0}}}^{m-1}+(n-2)\left(\beta-\gamma \cos n \phi_{0}\right) B_{0}^{n-2}\right]$ $\times\left(n \gamma B_{0}^{n-1} \cos n \phi_{0}\right) \eta$

Table 1

(i)

\section{-Conditions on Parametric-

Frequency Axcitation

$$
\omega^{2}-1>0 \quad \gamma-\beta>0
$$$$
\omega^{2}-1>0
$$$$
\gamma-\beta<0
$$

$\omega^{2}-1<0$

$\gamma-\beta>0$

$\omega^{2}-1<0$

$$
\gamma-\beta<0
$$

Phase
$\left(n \phi_{0}\right)$$\quad \begin{gathered}\text { Number of } \\ \text { singularities }\end{gathered}$

$\left(n \phi_{0}\right)$

0
$\pi$
0
$\pi$
0
$\pi$
0
$\pi$

1

1

1

2 or zero

0
0
0
Nature of singularities Center Saddle point Center Saddle point 1 saddle point 1 center

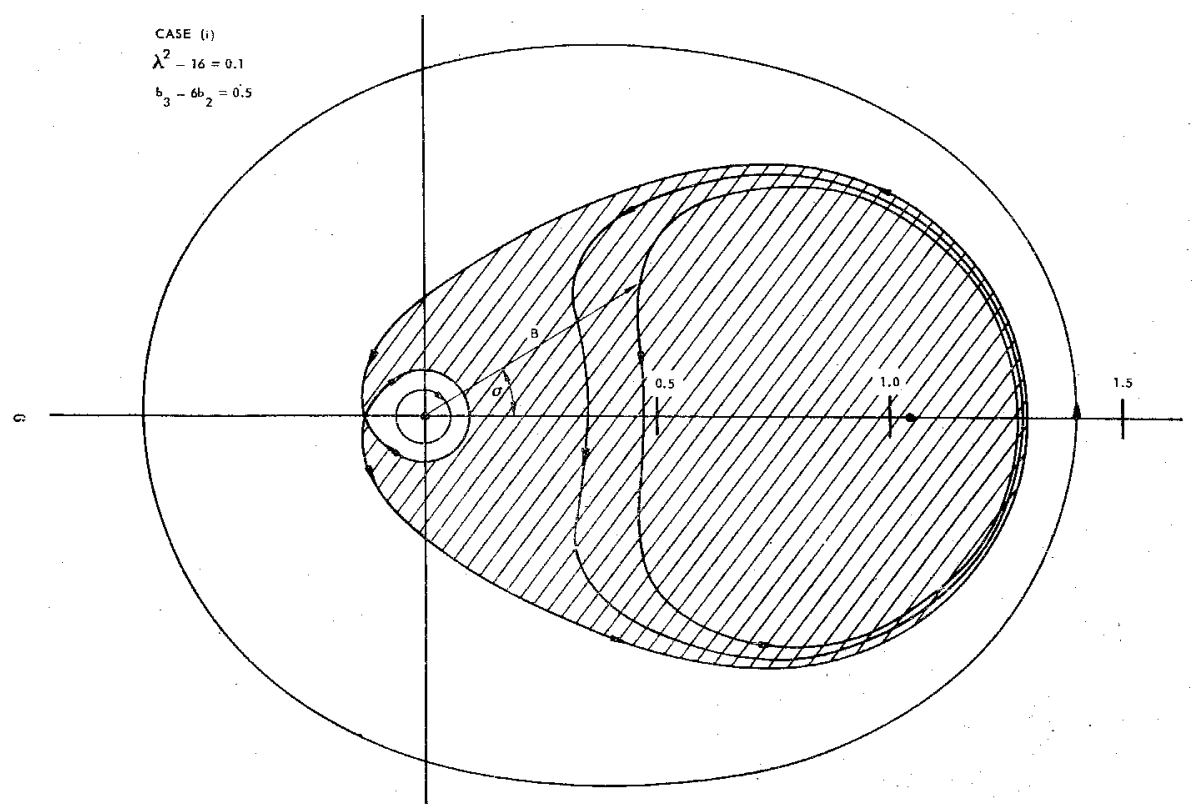

Fig. $1(a)$ Phase-plane diagram $\left(m=5, n=4, b_{1}=0.1, b_{2}=0.2\right)$ 


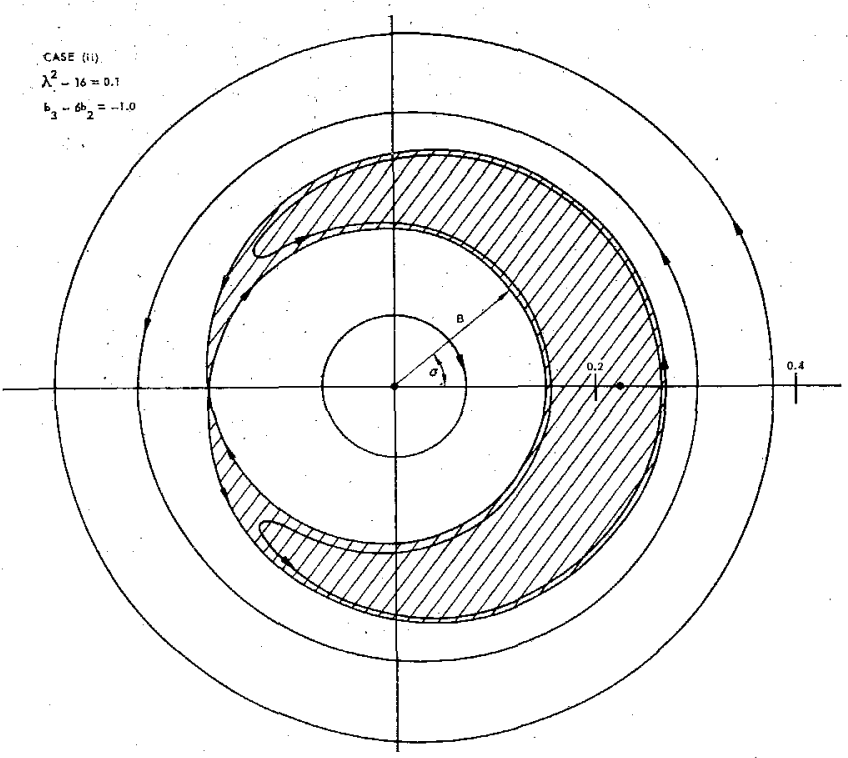

Fig. 1(b) Phase-plane diagram $\left(m=5, n=4, b_{1}=0.1, b_{2}=0.2\right)$

Let

$$
\eta=e^{\mu t}
$$

and

$$
F(B) \equiv \alpha B^{m-1}+\left(\beta-\gamma \cos n \phi_{0}\right) B^{n-2}+\frac{1-\omega^{2}}{2 \omega}
$$

Equation (22) can then be written

$$
\mu^{2}=-n \gamma B_{0}{ }^{n-1} F^{\prime}\left(B_{0}\right) \cos n \phi_{0}
$$

where $F^{\prime}\left(B_{0}\right) \equiv d F / d B$ at $B=B_{0}$. Since $n \gamma B_{0}{ }^{n-1}$ is always positive, the sign of $\mu^{2}$ depends on the signs of $F^{\prime}\left(B_{0}\right)$ and $\cos n \phi_{0}$. If $\mu^{2}$ is positive, $\mu$ is real and the trajectories in the phase plane diverge away from the singularity, indicating the singularity is a saddle point. If $\mu^{2}$ is negative, $\mu$ is imaginary and both $\xi$ and $\eta$ are bounded, showing the singularity is a center. Thus, the nature of the singularities can be studied using equation (25). The sign of $F^{\prime}\left(B_{0}\right)$ can be found by a sketch of the function $F(B)$ against $B$, noting that $F\left(B_{0}\right)=0$.

From a plot of $F(B)$ against $B$ it can be shown that value of $B_{0}$ for case (i) with phase angle $n \phi_{0}=0$ is larger than the corresponding value of $B_{0}$ for case (ii). In both cases, the singularities are centers. In case (iii) of Table 1 , with phase angle $n \phi_{0}=0$, the number of singularities depends on the relative magnitude $\left(1-\omega^{2}\right) / 2 \omega$ and $(\gamma-\beta)$. If $\left(1-\omega^{2}\right) / 2 \omega$ is small and $(\gamma-\beta)$ is large, equation (17) has two positive real roots; the smaller root corresponds to a saddle point and the larger root corresponds to a center. Conversely, if $\left(1-\omega^{2}\right) / 2 \omega$ is large and $(\gamma-\beta)$ is small, no real root exists. The nature of the singularity at the origin, $B_{0}=0$, can be shown to be a center for all the cases considered in Table 1.

It is known that no positive statement can be made on the stability of a singularity when a singularity is found to be a center under infinitesimal perturbational analysis. The full nonlinear equation has to be used to determine the true character of the singularity. Using equation (12) to plot the trajectories in the $(B-\phi)$-plane, it is found that the centers as determined from the infinitesimal perturbational analysis are true centers. A summary of the nature of the singularities is shown in Table 1 . It is convenient to plot $B$ against $\sigma$ where $\sigma \equiv n \phi$ instead of $B$ against $\phi$ so that the general features of the trajectories plotted will apply to all values of $n$. Plots of the trajectories for the four cases considered in Table 1 are shown in Figs. $1(a), 1(b),(2 a)$, and $2(b)$ for special values of $m=5$, and $n=4$.

\section{Example}

Consider the case $m=5, n=4$. Equation (1) becomes

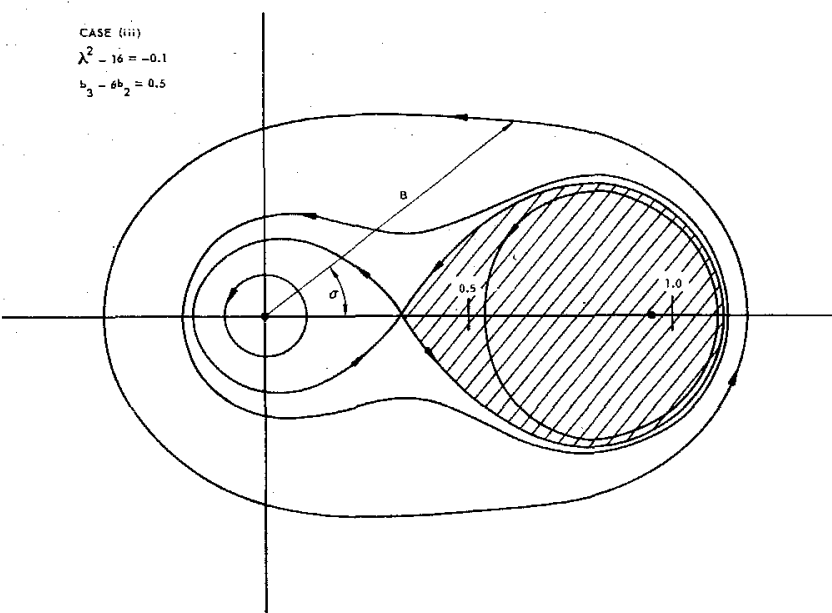

Fig. 2(a) Phase-plane diagram $\left(m=5, n=4, b_{1}=0.1, b_{2}=0.2\right)$

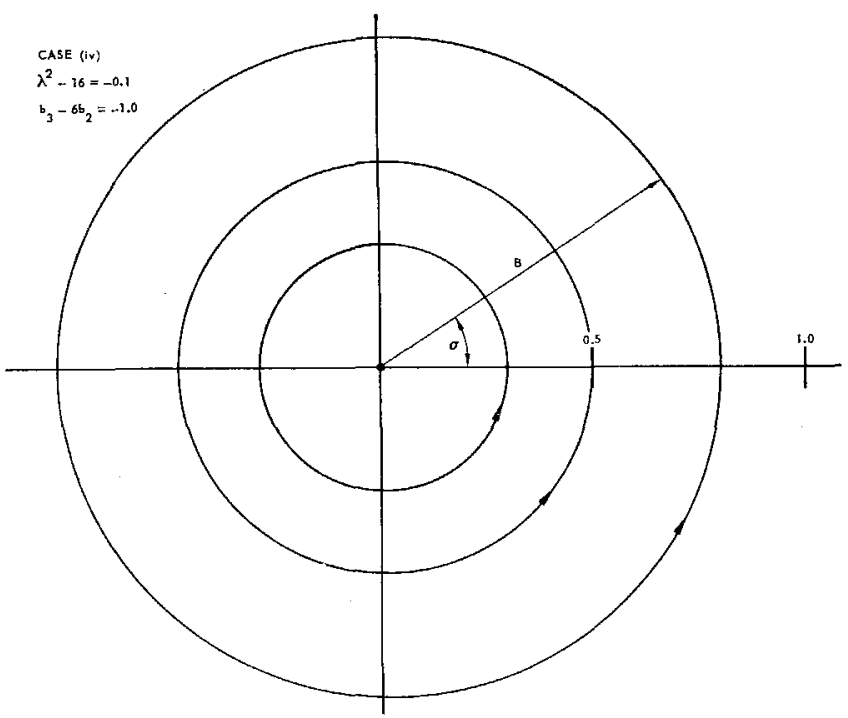

Fig. 2(b) Phase-plane diagram $\left(m=5, n=4, b_{1}=0.1, b_{2}=0.2\right)$

$$
\ddot{x}+x+b_{1} x^{5}+b_{2} x^{3}-b_{3} x^{3} \cos \lambda t=0
$$

The singularities in the $(B-\sigma)$-phase plane excluding the origin are given by the equations

$$
4 \phi_{0}=0 \text { or } \pi
$$

and

$$
10 b_{1} B_{0}{ }^{4}+2\left(6 b_{2}-b_{3} \cos 4 \phi_{0}\right) B_{0}{ }^{2}-\left(\lambda^{2}-16\right)=0
$$

Let $\lambda^{2}-16 \equiv \epsilon$ and $|\epsilon| \ll 1$. This corresponds to the parametric exciting frequency being a close multiple of the frequency of the mode to be excited. Solving equation (28),

$$
\begin{aligned}
B_{0}{ }^{2}=\frac{1}{10 b_{1}}\left\{\left(b_{3} \cos 4 \phi_{0}\right.\right. & \left.-6 b_{2}\right) \\
& \left. \pm\left[\left(b_{3} \cos 4 \phi_{0}-6 b_{2}\right)^{2}+10 b_{1} \epsilon\right]^{1 / 2}\right\}
\end{aligned}
$$

The distribution of singularities can be seen from equation (29).

When $\epsilon$ is negative but small, the expression under the squareroot sign is positive. If $\left(b_{3} \cos 4 \phi_{0}-6 b_{2}\right)>0$, two positive roots exist and can be approximated to

$$
\frac{-\epsilon}{2\left(b_{3}-6 b_{2}\right)}
$$

and

$$
\left(b_{3}-6 b_{2}\right) / 5 b_{1} .
$$

If $\left(b_{3} \cos 4 \phi_{0}-6 b_{2}\right)<0$, no positive real root exists. 


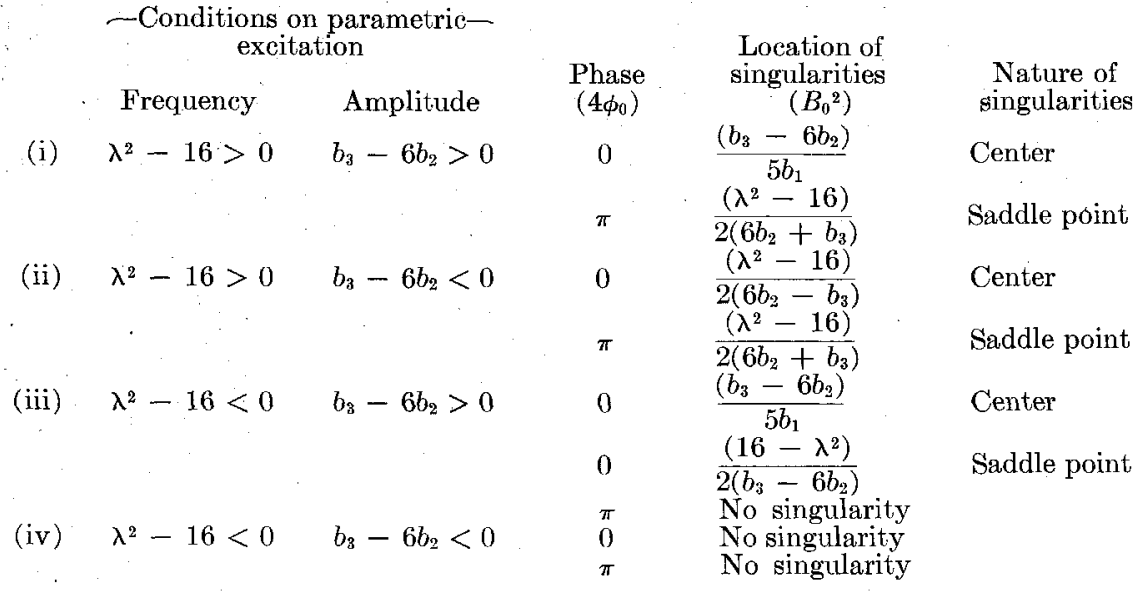

When the parametric exciting frequency is close to but larger than four times the frequency of the system, $\epsilon$ is positive and it can be seen from equation (29) that there always exists a positive root of $B_{0}$. The root has a larger value when $\left(b_{3} \cos 4 \phi_{0}-6 b_{2}\right)$ is positive. The approximate location and the nature of the singularities are given in Table 2. The equation of the trajectory in the phase plane takes the form

$$
\frac{10}{3} b_{1} B^{6}+\left(6 b_{2}-b_{3} \cos 4 \phi\right) B^{4}+\left(16-\lambda^{2}\right) B^{2}=16 \lambda K
$$

Plots of equation (30) with $b_{1}=0.1, b_{2}=0.2$ and different values of $b_{3}$ and $\lambda$ are shown in Figs. 1(a), 1(b), 2(a), and $2(b)$.

It can be seen that motions in all regions of the phase plane are amplitude stable, but the "phase" $\sigma$ is stable only at the shaded regions. The net change of $\sigma$ in such regions after one cycle is zero, while the net change is $2 \pi$ in the unshaded regions. Recalling $\sigma=n \phi$, it can be deduced from equation (2) that a steadystate subharmonic solution of order $1 / 4$ is possible within the shaded regions.

It is interesting to note that the area of the shaded region and also the singularity inside the shaded region are highly dependent on the amplitude of the parametric excitation. For the exciting amplitude $b_{3}$ less than the critical value of $6 b_{2}$, no shaded area exists in the cases $\lambda^{2}-16<0$, and the shaded area is small in the case $\lambda^{2}-16>0$. The center inside the region is close to the origin in the latter case. On the other hand, when the exciting amplitude exceeds the critical value, shaded regions exist under both frequency conditions and the distance of the center from the origin is large. Large amplitude subharmonic motion is possible under such circumstances. The trajectories in the $(B-\sigma)$-phase plane for general values of $m$ and $n$ have similar features to those as shown in Figs. $1(a), 1(b), 2(a)$, and $2(b)$. Therefore, a steady state subharmonic solution of order $1 / n$ is possible within the shaded regions in the general case.

The foregoing analysis agrees with experimental observations on testing of the thin-walled beams; that subharmonic behavior was observed only when a higher mode was in resonance so that the parametric excitation amplitude was large; and also that the exciting frequency was a multiple or near multiple of the frequency of the mode to be excited parametrically.

Equation (26) was also solved on an analog computer under different parametric-excitation conditions. The results agreed well with the analysis. A typical result is shown in Fig. 3 . The trace with higher frequency is the parametric excitation and the trace with lower frequency is the response of the system. Superposition of the two traces as shown illustrates the existence of a subharmonic solution of order $1 / 4$, as predicted from the analysis.

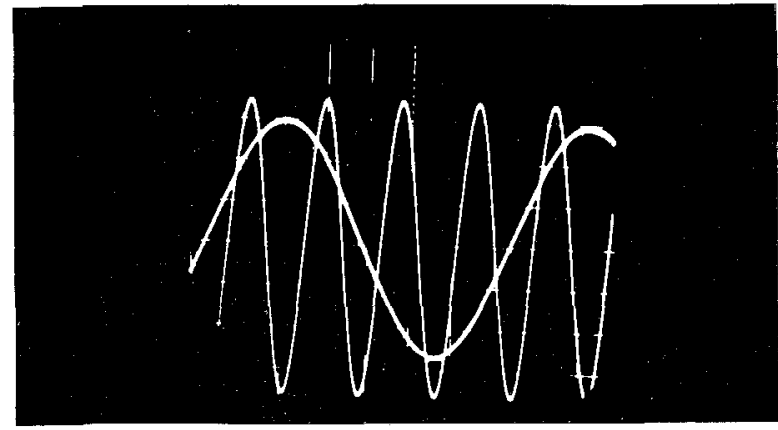

Fig. 3 Analog-compữer solution of equation (26)

It should be pointed out that the system represented by equation (1) can have subharmonic solutions of lower order under suitable parametric excitation. But subharmonic of order $1 / n$ is the highest order subharmonic the system can produce. In the experiment on the analog computer, a sibharmonic solution of $1 / 2$ was also observed under proper parametric excitation.

\section{Remarks}

The foregoing analysis serves two purposes. First, it presents a possible mechanism for what is observed in the experiment of thin-walled beams of open section. Second, it points to the fact that in nonlinear multidegree-of-freedom systems, very high order subharmonics can occur. When one mode of the system is excited externally, some other mode can be excited parametrically to execute subharmonic motion. The linearization of the parametrically excited equation will automatically exclude the possibility of predicting such high-order subharmonics.

\section{References}

1 J. J. Stoker, Nonlinear Vibrations, Interscience Publishers, Inc. New York, N. Y., 1950.

2 E. Sevin, "On the Parametric Excitation of Pendulum-Type Vibration Absorber," Journat of Applied Mechanios, vol. 28 Trans. ASME, vol. 83, Series E, 1961, pp. 330-334.

3 C.S. Hsu, "On the Parametric Excitation of a Dynamic System Having Multiple Degrees of Freedom," Journal of APpiIed MEChanics, vol. 30, Thans. ASME, vol. 85, Series E, 1963, pp. 367-372.

4 W. K. Tso, "Subharmonic Vibrations of Thin-Walled Elastic Beams," to be published in Experimental Mechanies.

5 W. K. Tso, "Dynamics of Thin-Walled Beams of Open Section," Dynamics Laboratory Report, California Institute of Technology, Pasadena, Calif., 1964.

6 N. W. McLachlan, Ordinary Non-Linear Differential Equations in Engineering and Physical Science, Oxford University Press. second edition, 1956 . 